\title{
Some basic properties of idempotent matrices
}

\author{
Alaa A. Hammodat Ali A. Bilal Akram S. Mohammed \\ Dept. of Math. \\ Dept. of Math. \\ Dept. of Math. \\ College of Education \\ College of Education \\ College of Science \\ University of Mosul \\ University of Mosul \\ University of Tikrit
}

Received

25 / 12 / 2006
Accepted

03 / 10 / 2007

\section{الخلاصة}

في هذا البهث قمنا بعض الخواص للمصفوفت المتحاية المتبادلة صفرياً وأعطينا

بعض الخواص للمصفوفلت غير المفردة.

\begin{abstract}
In this paper we give some properties of the zero commut idempotent matrices , and give some properties of non-singular matrices .
\end{abstract}




\section{Introduction}

Throughout, this paper all matrices considered are square and commutative unless other wise stated.

In [2], Koliha, Racocevic and Straskraba present new results on the invertibility of the sum of projectors, new relations between the nonsingularity of the difference and the sum of projectors, and present a simple proof of the invertibility of $\mathrm{n} \times \mathrm{n}$ matrix A exists by showing that $\mathrm{N}(\mathrm{A})=\{0\}$. In this paper we present some basic properties of an idempotent matrices and relations between the range and the null spaces and give some results on the non-singularity of the difference and sum of idempotent matrices. We recall that;

1) A matrix $A$ is said to be idempotent if $A^{2}=A$.

2) A two matrices $A$ and $B$ are said to be zero commut if $A B=B A=0$.

3) A null space of a matix $A$ is the set of all solutions to the equation $\mathrm{A} \overrightarrow{\mathbf{x}}=0, \operatorname{Null}(\mathrm{A})=\left\{\overrightarrow{\mathbf{x}} \in \mathrm{R}^{\mathrm{n}}: \mathrm{A} \overrightarrow{\mathbf{x}}=0\right\}$, and we denote that $\mathrm{N}(\mathrm{A})$.

4) A range space of a matrix $A$ is the set of all solutions to the equation $\mathrm{A} \overrightarrow{\mathbf{x}}=\overrightarrow{\mathbf{x}}$, and we denote that $\mathrm{R}(\mathrm{A})$.

\section{Idempotent matrices} matrices.

In this section we give some basic properties of the idempotent

\section{Lemma 2.1 :}

\section{Proof:}

If $\mathrm{A}$ is idempotent matrix, then (I-A) is idempotent .

Trivial.

\section{Proposition 2.2 [2] :}

If $A$ is a matrix, then $N(A)=R(I-A)$, also $\mathrm{N}(\mathrm{I}-\mathrm{A})=\mathrm{R}(\mathrm{A})$.

\section{Proposition 2.3 :}

If $A$ is a matrix, then $\mathrm{R}(\mathrm{A}) \cap \mathrm{N}(\mathrm{A})=\{0\}$.

\section{Proof:}

Let $\overrightarrow{\mathbf{x}} \in \mathrm{R}(\mathrm{A}) \cap \mathrm{N}(\mathrm{A})$, then $\overrightarrow{\mathbf{x}} \in \mathrm{R}(\mathrm{A})$ and $\overrightarrow{\mathbf{x}} \in \mathrm{N}(\mathrm{A})$.

Then $\overrightarrow{\mathbf{x}}=\mathrm{A} \overrightarrow{\mathbf{x}}$ and $A \overrightarrow{\mathbf{x}}=0$, so, $\overrightarrow{\mathbf{x}}=0$.

Hence $\mathrm{R}(\mathrm{A}) \cap \mathrm{N}(\mathrm{A})=\{0\}$.

\section{Proposition 2.4 :}

\section{Proof:}

If $A$ and $B$ be are idempotent matrices, then $R(A) \cap R[B(I-A)]=\{0\}$.

Let $\overrightarrow{\mathbf{y}} \in R(A) \cap R[B(I-A)]$, then $\overrightarrow{\mathbf{y}}=A \overrightarrow{\mathbf{y}}$ and $\overrightarrow{\mathbf{y}}=B(I-A) \overrightarrow{\mathbf{y}}$. 
So $A \overrightarrow{\mathbf{y}}=A B(I-A) \overrightarrow{\mathbf{y}}=A B \overrightarrow{\mathbf{y}}-A B A \overrightarrow{\mathbf{y}}=0$.

But $A \overrightarrow{\mathbf{y}}=\overrightarrow{\mathbf{y}}$, so $\overrightarrow{\mathbf{y}}=0$.

Hence $\mathrm{R}(\mathrm{A}) \cap \mathrm{R}[\mathrm{B}(\mathrm{I}-\mathrm{A})]=\{0\}$.

\section{Proposition 2.5:}

If $A$ and $B$ be are matrices, with $A B=A$ and $B A=B$. Then $N(A)=N(B)$.

Proof:

Let $\overrightarrow{\mathbf{x}} \in \mathrm{N}(\mathrm{A})$, then $\mathrm{A} \overrightarrow{\mathbf{x}}=0$, so $\mathrm{BA} \overrightarrow{\mathbf{x}}=0$, but $\mathrm{BA}=\mathrm{B}$. Therefore $\mathrm{B} \overrightarrow{\mathbf{x}}=0$ and $\overrightarrow{\mathbf{x}} \in \mathrm{N}(B)$. Hence $\mathrm{N}(\mathrm{A}) \subseteq \mathrm{N}(\mathrm{B})$.

Similarly $\mathrm{N}(\mathrm{B}) \subseteq \mathrm{N}(\mathrm{A})$.

Therefore $\mathrm{N}(\mathrm{A})=\mathrm{N}(\mathrm{B})$.

\section{Proposition 2.6 :}

If $A$ and $B$ be are idempotent matrices, then $R(A B)=R(A) \cap R(B)$.

\section{Proof:}

Let $\overrightarrow{\mathbf{x}} \in \mathrm{R}(\mathrm{AB})$, then $\overrightarrow{\mathbf{x}}=\mathrm{AB} \overrightarrow{\mathbf{x}}$.

So (I-A) $\overrightarrow{\mathbf{x}}=(\mathrm{I}-\mathrm{A}) \mathrm{AB} \overrightarrow{\mathbf{x}}=0$.

Hence $\overrightarrow{\mathbf{x}} \in \mathrm{N}(\mathrm{I}-\mathrm{A})=\mathrm{R}(\mathrm{A})$ [by Proposition 2.2] .

Similarly $\overrightarrow{\mathbf{x}} \in R(B)$, so $\overrightarrow{\mathbf{x}} \in \mathrm{R}(\mathrm{A}) \cap \mathrm{R}(\mathrm{B})$.

Therefore $\mathrm{R}(\mathrm{AB}) \subseteq \mathrm{R}(\mathrm{A}) \cap \mathrm{R}(\mathrm{B})$.

Now, let $\overrightarrow{\mathbf{y}} \in R(A) \cap R(B)$, then $\overrightarrow{\mathbf{y}}=A \overrightarrow{\mathbf{y}}$ and $\overrightarrow{\mathbf{y}}=B \overrightarrow{\mathbf{y}}$.

So $A \overrightarrow{\mathbf{y}}=B \overrightarrow{\mathbf{y}}$. That is $A \overrightarrow{\mathbf{y}}=A B \overrightarrow{\mathbf{y}}$,

but $\overrightarrow{\mathbf{y}}=A \overrightarrow{\mathbf{y}}$, therefore $\overrightarrow{\mathbf{y}}=A B \overrightarrow{\mathbf{y}} \in R(A B)$.

So $\mathrm{R}(\mathrm{A}) \cap \mathrm{R}(\mathrm{B}) \subseteq \mathrm{R}(\mathrm{AB})$.

Hence $R(A B)=R(A) \cap R(B)$.

\section{Proposition 2.7 :}

If $A$ and $B$ be are idempotent matrices, and $R(A) \cap R(B)=\{0\}$, then

$\mathrm{N}(\mathrm{A}-\mathrm{B})=\mathrm{N}(\mathrm{A}) \cap \mathrm{N}(\mathrm{B})$.

\section{Proof:}

Let $\overrightarrow{\mathbf{x}} \in \mathrm{N}(\mathrm{A}-\mathrm{B})$, then $(\mathrm{A}-\mathrm{B}) \overrightarrow{\mathbf{x}}=0$ and $\mathrm{A} \overrightarrow{\mathbf{x}}=\mathrm{B} \overrightarrow{\mathbf{x}}$.

So $A \overrightarrow{\mathbf{x}}=A^{2} \overrightarrow{\mathbf{x}}=A B \overrightarrow{\mathbf{x}}=B A \overrightarrow{\mathbf{x}}$.

Now, from $A \overrightarrow{\mathbf{x}}=B A \overrightarrow{\mathbf{x}}$ we get $(B-I) A \overrightarrow{\mathbf{x}}=0$, so $A \overrightarrow{\mathbf{x}} \in \mathrm{N}(\mathrm{B}-\mathrm{I})=\mathrm{R}(\mathrm{B})$

[by Proposition2.2], but $A \overrightarrow{\mathbf{x}}=B \overrightarrow{\mathbf{x}}$, so $B \overrightarrow{\mathbf{x}} \in R(B)$.

Similarly from $B \overrightarrow{\mathbf{x}}=A B \overrightarrow{\mathbf{x}}$ we get $A \overrightarrow{\mathbf{x}}, B \overrightarrow{\mathbf{x}} \in R(A)$.

So $A \overrightarrow{\mathbf{x}}, B \overrightarrow{\mathbf{x}} \in R(A) \cap R(B)=\{0\}$, therefore $\overrightarrow{\mathbf{x}} \in \mathrm{N}(A)$.

Also, $\overrightarrow{\mathbf{x}} \in \mathrm{N}(B)$, so $\overrightarrow{\mathbf{x}} \in \mathrm{N}(A) \cap \mathrm{N}(B)$.

Hence $\mathrm{N}(\mathrm{A}-\mathrm{B}) \subseteq \mathrm{N}(\mathrm{A}) \cap \mathrm{N}(\mathrm{B})$.

Now, let $\overrightarrow{\mathbf{y}} \in \mathrm{N}(A) \cap N(B)$, then $A \overrightarrow{\mathbf{y}}=0$ and $B \overrightarrow{\mathbf{y}}=0$.

So (A-B) $\overrightarrow{\mathbf{y}}=0$, therefore $\overrightarrow{\mathbf{y}} \in \mathrm{N}(\mathrm{A}-\mathrm{B})$.

So $\mathrm{N}(\mathrm{A}) \cap \mathrm{N}(\mathrm{B}) \subseteq \mathrm{N}(\mathrm{A}-\mathrm{B})$.

Hence $N(A-B)=N(A) \cap N(B)$. 


\section{Proposition 2.8 :}

If $A$ and $B$ be are idempotent matrices, then $R[B(I-A)]=N(A) \cap R(B)$.

\section{Proof:}

Let $\overrightarrow{\mathbf{x}} \in \mathrm{R}[\mathrm{B}(\mathrm{I}-\mathrm{A})]$, then $\overrightarrow{\mathbf{x}}=\mathrm{B}(\mathrm{I}-\mathrm{A}) \overrightarrow{\mathbf{x}}$.

So $A \overrightarrow{\mathbf{x}}=A B(I-A) \overrightarrow{\mathbf{x}}=A B \overrightarrow{\mathbf{x}}-A B A \overrightarrow{\mathbf{x}}=0$.

So $\overrightarrow{\mathbf{x}} \in \mathrm{N}(\mathrm{A})$.

Also, (I-B) $\overrightarrow{\mathbf{x}}=(I-B) B(I-A) \overrightarrow{\mathbf{x}}=0$, so $\overrightarrow{\mathbf{x}}=B \overrightarrow{\mathbf{x}}$, therefore $\overrightarrow{\mathbf{x}} \in \mathrm{R}(\mathrm{B})$.

So $\overrightarrow{\mathbf{x}} \in \mathrm{N}(\mathrm{A}) \cap \mathrm{R}(\mathrm{B})$.

Hence $\mathrm{R}[\mathrm{B}(\mathrm{I}-\mathrm{A})] \subseteq \mathrm{N}(\mathrm{A}) \cap \mathrm{R}(\mathrm{B})$.

Now, let $\overrightarrow{\mathbf{y}} \in \mathrm{N}(\mathrm{A}) \cap \mathrm{R}(\mathrm{B})$ then $\mathrm{A} \overrightarrow{\mathbf{y}}=0$ and $\overrightarrow{\mathbf{y}}=\mathrm{B} \overrightarrow{\mathbf{y}}$.

So $A \overrightarrow{\mathbf{y}}=A B \overrightarrow{\mathbf{y}}=0$.

Now, B(I-A) $\overrightarrow{\mathbf{y}}=B(I-A) B \overrightarrow{\mathbf{y}}=B \overrightarrow{\mathbf{y}}-B A B \overrightarrow{\mathbf{y}}$.

Therefore $B(I-A) \overrightarrow{\mathbf{y}}=B \overrightarrow{\mathbf{y}}=\overrightarrow{\mathbf{y}}$, so $\overrightarrow{\mathbf{y}} \in R[B(I-A)]$.

Hence $\mathrm{N}(\mathrm{A}) \cap \mathrm{R}(\mathrm{B}) \subseteq \mathrm{R}[\mathrm{B}(\mathrm{I}-\mathrm{A})]$.

Therefore $\mathrm{R}[\mathrm{B}(\mathrm{I}-\mathrm{A})]=\mathrm{N}(\mathrm{A}) \cap \mathrm{R}(\mathrm{B})$.

\section{Proposition 2.9 :}

If $A$ and $B$ be are idempotent matrices, then $R(A)=R(B)$ if and only if $\mathrm{N}(\mathrm{A})=\mathrm{N}(\mathrm{B})$.

\section{Proof:} $\mathrm{B} \overrightarrow{\mathbf{x}}=0$.

Let $R(A)=R(B)$, then $A \overrightarrow{\mathbf{x}}=B \overrightarrow{\mathbf{x}}$ and let $\overrightarrow{\mathbf{x}} \in \mathrm{N}(\mathrm{A})$, then $A \overrightarrow{\mathbf{x}}=0$, so

Then $\overrightarrow{\mathbf{x}} \in \mathrm{N}(\mathrm{B})$, therefore $\mathrm{N}(\mathrm{A}) \subseteq \mathrm{N}(\mathrm{B})$.

Similarly $\mathrm{N}(\mathrm{B}) \subseteq \mathrm{N}(\mathrm{A})$, so $\mathrm{N}(\mathrm{A})=\mathrm{N}(\mathrm{B})$.

Now, let $N(A)=N(B)$ and let $\overrightarrow{\mathbf{y}} \in \mathrm{R}(A)$, then $\overrightarrow{\mathbf{y}}=\mathrm{A} \overrightarrow{\mathbf{y}}$.

So $B \overrightarrow{\mathbf{y}}=B A \overrightarrow{\mathbf{y}}=A B \overrightarrow{\mathbf{y}}$.

From $B(I-B) \overrightarrow{\mathbf{y}}=0$, we get $(I-B) \overrightarrow{\mathbf{y}} \in \mathrm{N}(B)=N(A)$ and $A(I-B) \overrightarrow{\mathbf{y}}=0$.

So $A \overrightarrow{\mathbf{y}}=A B \overrightarrow{\mathbf{y}}$, but $\overrightarrow{\mathbf{y}}=A \overrightarrow{\mathbf{y}}$ and $B \overrightarrow{\mathbf{y}}=A B \overrightarrow{\mathbf{y}}$.

Therefore $\overrightarrow{\mathbf{y}}=B \overrightarrow{\mathbf{y}}$ and $\overrightarrow{\mathbf{y}} \in R(B)$, so $R(A) \subseteq R(B)$.

Similarly $\mathrm{R}(\mathrm{B}) \subseteq \mathrm{R}(\mathrm{A})$.

Hence $R(A)=R(B)$.

\section{Proposition 2.10 :}

If $A$ and $B$ be are idempotent matrices, then $N(A) \cap N(B)=N(A+B-A B)$.

\section{Proof:}

Let $\overrightarrow{\mathbf{x}} \in \mathrm{N}(\mathrm{A}) \cap \mathrm{N}(\mathrm{B})$, then $\mathrm{A} \overrightarrow{\mathbf{x}}=0$ and $\mathrm{B} \overrightarrow{\mathbf{x}}=0$, so $\mathrm{AB} \overrightarrow{\mathbf{x}}=0$.

Therefore $(A+B-A B) \overrightarrow{\mathbf{x}}=0$ and $\overrightarrow{\mathbf{x}} \in \mathrm{N}(\mathrm{A}+\mathrm{B}-\mathrm{AB})$.

So $\mathrm{N}(\mathrm{A}) \cap \mathrm{N}(\mathrm{B}) \subseteq \mathrm{N}(\mathrm{A}+\mathrm{B}-\mathrm{AB})$.

Now, let $\overrightarrow{\mathbf{y}} \in N(A+B-A B)$, then $(A+B-A B) \overrightarrow{\mathbf{y}}=0$, so $A \overrightarrow{\mathbf{y}}+B \overrightarrow{\mathbf{y}}=A B \overrightarrow{\mathbf{y}}$.

Hence $A^{2} \overrightarrow{\mathbf{y}}+A B \overrightarrow{\mathbf{y}}=A^{2} B \overrightarrow{\mathbf{y}}$. So $A \overrightarrow{\mathbf{y}}=0$ and $\overrightarrow{\mathbf{y}} \in N(A)$. 
Similarly $B \overrightarrow{\mathbf{y}}=0$ and $\overrightarrow{\mathbf{y}} \in \mathrm{N}(B)$.

So $\overrightarrow{\mathbf{y}} \in \mathrm{N}(\mathrm{A}) \cap \mathrm{N}(\mathrm{B})$.

Hence $N(A+B-A B) \subseteq N(A) \cap N(B)$.

Therefore $\mathrm{N}(\mathrm{A}) \cap \mathrm{N}(\mathrm{B})=\mathrm{N}(\mathrm{A}+\mathrm{B}-\mathrm{AB})$.

\section{Basic properties of zero commut idempotent matrices.}

In this section we present some basic properties of the zero commut idempotent matrices.

\section{Proposition 3.1 :}

If $\mathrm{A}$ and $\mathrm{B}$ be are zero commut idempotent matrices, then $\mathrm{N}(\mathrm{A}-\mathrm{B})=\mathrm{N}(\mathrm{A}) \cap \mathrm{N}(\mathrm{B})$.

\section{Proof:}

Let $\overrightarrow{\mathbf{x}} \in \mathrm{N}(\mathrm{A}-\mathrm{B})$, then $(\mathrm{A}-\mathrm{B}) \overrightarrow{\mathbf{x}}=0$ and $\mathrm{A} \overrightarrow{\mathbf{x}}=\mathrm{B} \overrightarrow{\mathbf{x}}$.

So $A \overrightarrow{\mathbf{x}}=A B \overrightarrow{\mathbf{x}}=0$.

Hence $\overrightarrow{\mathbf{x}} \in \mathrm{N}(\mathrm{A})$.

Similarly $\overrightarrow{\mathbf{x}} \in \mathrm{N}(\mathrm{B})$, so $\overrightarrow{\mathbf{x}} \in \mathrm{N}(\mathrm{A}) \cap \mathrm{N}(\mathrm{B})$.

Hence $\mathrm{N}(\mathrm{A}-\mathrm{B}) \subseteq \mathrm{N}(\mathrm{A}) \cap \mathrm{N}(\mathrm{B})$.

Now, let $\overrightarrow{\mathbf{y}} \in \mathrm{N}(A) \cap N(B)$, then $A \overrightarrow{\mathbf{y}}=0$ and $B \overrightarrow{\mathbf{y}}=0$, so $(A-B) \overrightarrow{\mathbf{y}}=0$.

Therefore $\overrightarrow{\mathbf{y}} \in \mathrm{N}(A-B)$, so $\mathrm{N}(A) \cap \mathrm{N}(\mathrm{B}) \subseteq \mathrm{N}(A-B)$.

Hence $N(A-B)=N(A) \cap N(B)$.

\section{Proposition 3.2 :}

If $\mathrm{A}$ and $\mathrm{B}$ be are zero commut idempotent matrices, then $\mathrm{N}(\mathrm{A}+\mathrm{B})=\mathrm{N}(\mathrm{A}) \cap \mathrm{N}(\mathrm{B})$.

Proof:

Similar to the proof of proposition 3.1

\section{Corollary 3.3 :}

If $\mathrm{A}$ and $\mathrm{B}$ be are zero commut idempotent matrices, then $\mathrm{N}(\mathrm{A}+\mathrm{B})=\mathrm{N}(\mathrm{A}-\mathrm{B})$.

\section{Proposition 3.4 :}

If $\mathrm{A}$ and $\mathrm{B}$ be are zero commut idempotent matrices, then $\mathrm{R}(\mathrm{A}+\mathrm{B})=\mathrm{R}(\mathrm{A})+\mathrm{R}(\mathrm{B})$.

\section{Proof:}

$$
\text { Let } \overrightarrow{\mathbf{y}}=\overrightarrow{\mathbf{y}}_{1}+\overrightarrow{\mathbf{y}}_{2} \in \mathrm{R}(\mathrm{A})+\mathrm{R}(\mathrm{B}),
$$

where $\overrightarrow{\mathbf{y}}_{1} \in \mathrm{R}(\mathrm{A})$ and $\overrightarrow{\mathbf{y}}_{2} \in \mathrm{R}(\mathrm{B})$.

$$
\begin{aligned}
\text { So }(A+B)\left(\overrightarrow{\mathbf{y}}_{1}+\overrightarrow{\mathbf{y}}_{2}\right)= & A \overrightarrow{\mathbf{y}}_{1}+\mathrm{A} \overrightarrow{\mathbf{y}}_{2}+\mathrm{B} \overrightarrow{\mathbf{y}}_{1}+\mathrm{B} \overrightarrow{\mathbf{y}}_{2} \\
& =\overrightarrow{\mathbf{y}}_{1}+\overrightarrow{\mathbf{y}}_{2}+\mathrm{A} \overrightarrow{\mathbf{y}}_{2}+\mathrm{B} \overrightarrow{\mathbf{y}}_{1}-0 \\
& =\overrightarrow{\mathbf{y}}_{1}+\overrightarrow{\mathbf{y}}_{2}+\mathrm{A} \overrightarrow{\mathbf{y}}_{2}+\mathrm{B} \overrightarrow{\mathbf{y}}_{1}-\mathrm{AB}\left(\overrightarrow{\mathbf{y}}_{1}+\overrightarrow{\mathbf{y}}_{2}\right)
\end{aligned}
$$




$$
\begin{aligned}
& =\overrightarrow{\mathbf{y}}_{1}+\overrightarrow{\mathbf{y}}_{2}+\mathrm{A}\left(\overrightarrow{\mathbf{y}}_{2}-\mathrm{B} \overrightarrow{\mathbf{y}}_{2}\right)+\mathrm{B}\left(\overrightarrow{\mathbf{y}}_{1}-\mathrm{A} \overrightarrow{\mathbf{y}}_{1}\right) \\
& =\overrightarrow{\mathbf{y}}_{1}+\overrightarrow{\mathbf{y}}_{2}=\overrightarrow{\mathbf{y}} .
\end{aligned}
$$

Therefore $\overrightarrow{\mathbf{y}}=\overrightarrow{\mathbf{y}}_{1}+\overrightarrow{\mathbf{y}}_{2} \in \mathrm{R}(\mathrm{A}+\mathrm{B})$, so $\mathrm{R}(\mathrm{A})+\mathrm{R}(\mathrm{B}) \subseteq \mathrm{R}(\mathrm{A}+\mathrm{B})$.

Now, let $\overrightarrow{\mathbf{x}} \in R(A+B)$, then $\overrightarrow{\mathbf{x}}=(A+B) \overrightarrow{\mathbf{x}}=A \overrightarrow{\mathbf{x}}+B \overrightarrow{\mathbf{x}}$.

Let $\overrightarrow{\mathbf{x}}_{1}=A \overrightarrow{\mathbf{x}}$, then $A \overrightarrow{\mathbf{x}}_{1}=A \overrightarrow{\mathbf{x}}=\overrightarrow{\mathbf{x}}_{1}$, so $\overrightarrow{\mathbf{x}}_{1} \in \mathrm{R}(\mathrm{A})$.

Also, let $\overrightarrow{\mathbf{x}}_{2}=B \overrightarrow{\mathbf{x}}$, then $B \overrightarrow{\mathbf{x}}_{2}=B \overrightarrow{\mathbf{x}}=\overrightarrow{\mathbf{x}}_{2}$, so $\overrightarrow{\mathbf{x}}_{2} \in R(B)$.

Therefore $\overrightarrow{\mathbf{x}}=\overrightarrow{\mathbf{x}}_{1}+\overrightarrow{\mathbf{x}}_{2} \in \mathrm{R}(\mathrm{A})+\mathrm{R}(\mathrm{B})$, so $\mathrm{R}(\mathrm{A}+\mathrm{B}) \subseteq \mathrm{R}(\mathrm{A})+\mathrm{R}(\mathrm{B})$.

Hence $R(A+B)=R(A)+R(B)$.

\section{Corollary 3.5 :}

\section{Proof:}

If $\mathrm{A}$ is an idempotent matrix, then $\mathrm{R}^{\mathrm{n}}=\mathrm{R}(\mathrm{A})+\mathrm{N}(\mathrm{A})$.

Then $\mathrm{A}$ and $\mathrm{I}-\mathrm{A}$ are zero commut idempotent matrices .

Now, from [Proposition3.4] we get $R(A+I-A)=R(A)+R(I-A)$.

Hence $R(I)=R(A)+N(A)$.

Therefore $R^{n}=R(A)+N(A)$.

\section{Proposition 3.6 :}

\section{Proof:}

If $A$ is an idempotent matrix, then $R^{n}=R(A) \oplus N(A)$.

By [Corollary3.5] we get $\mathrm{R}^{\mathrm{n}}=\mathrm{R}(\mathrm{A})+\mathrm{N}(\mathrm{A})$, and by [Proposition2.3] we get $\mathrm{R}(\mathrm{A}) \cap \mathrm{N}(\mathrm{A})=\{0\}$. Hence $R^{n}=R(A) \oplus N(A)$.

\section{Basic properties of non-singular matrices.} matrices.

In this section we give some basic properties of a non-singular

\section{Remark:}

The only nonsingular idempotent matrix is identity matrix $\left(I_{n}\right)$. Every idempotent matrix (except $I_{n}$ ) is singular but a singular matrix may not be idempotent.

\section{Theorem 4.1 [1]:}

An $n \times n$ matrix $A$ over a number field $F$ has rank $n$ if and only if $\mathrm{A}^{-1}$ exists, that is, if and only if $\mathrm{A}$ is non-singular.

\section{Theorem 4.2 [2]:}

If $\mathrm{A}$ and $\mathrm{B}$ be are idempotent matrices, then the following conditions are equivalent:

1) A-B is non-singular.

2) $\mathrm{A}+\mathrm{B}$ and $\mathrm{I}-\mathrm{AB}$ are non-singular. 


\section{Proposition4.3:}

Let $\mathrm{A}$ and $\mathrm{B}$ be are idempotent matrices and $\mathrm{AB}$ is non-singular, then $\mathrm{N}(\mathrm{A}) \cap \mathrm{R}(\mathrm{B})=\{0\}$.

Proof:

Let $\overrightarrow{\mathbf{x}} \in \mathrm{N}(\mathrm{A}) \cap \mathrm{R}(\mathrm{B})$, then $\mathrm{A} \overrightarrow{\mathbf{x}}=0$ and $\overrightarrow{\mathbf{x}}=\mathrm{B} \overrightarrow{\mathbf{x}}$.

So $A \overrightarrow{\mathbf{x}}=A B \overrightarrow{\mathbf{x}}$. Hence $A B \overrightarrow{\mathbf{x}}=0$.

Therefore $\overrightarrow{\mathbf{x}} \in \mathrm{N}(\mathrm{AB})=\{0\}$ (since $\mathrm{AB}$ is non-singular), so $\overrightarrow{\mathbf{x}}=0$.

Hence $\mathrm{N}(\mathrm{A}) \cap \mathrm{R}(\mathrm{B})=\{0\}$.

\section{Theorem 4.4 :}

If $\mathrm{A}$ and $\mathrm{B}$ be are zero commut idempotent matrices, and $\mathrm{A}-\mathrm{B}$ is non-singular, then $R^{n}=R(A) \oplus R(B)$.

Proof:

Let $\overrightarrow{\mathbf{x}} \in \mathrm{R}(\mathrm{A}) \cap \mathrm{R}(\mathrm{B})$, then $\overrightarrow{\mathbf{x}} \in \mathrm{R}(\mathrm{A})$ and $\overrightarrow{\mathbf{x}} \in \mathrm{R}(\mathrm{B})$.

Therefore $\overrightarrow{\mathbf{x}}=A \overrightarrow{\mathbf{x}}$ and $\overrightarrow{\mathbf{x}}=B \overrightarrow{\mathbf{x}}$, so $A \overrightarrow{\mathbf{x}}=B \overrightarrow{\mathbf{x}}$, then $(A-B) \overrightarrow{\mathbf{x}}=0$.

Hence $\overrightarrow{\mathbf{x}} \in \mathrm{N}(\mathrm{A}-\mathrm{B})=0$ (since $A-\mathrm{B}$ is non-singular) .

So $\overrightarrow{\mathbf{x}}=0$, hence $\mathrm{R}(\mathrm{A}) \cap \mathrm{R}(\mathrm{B})=\{0\}$.

Since $(A-B)(I-A-B) \overrightarrow{\mathbf{x}}=0$, then $(I-A-B) \overrightarrow{\mathbf{x}} \in N(A-B)=0$,

and (I-A-B) $\overrightarrow{\mathbf{x}}=0$.Hence $\overrightarrow{\mathbf{x}}=A \overrightarrow{\mathbf{x}}+B \overrightarrow{\mathbf{x}} \in \mathrm{R}(\mathrm{A})+\mathrm{R}(\mathrm{B})$ by [Proposition3.4].

So $\mathrm{R}^{\mathrm{n}}=\mathrm{R}(\mathrm{A})+\mathrm{R}(\mathrm{B})$.

Hence $R^{n}=R(A) \oplus R(B)$.

\section{Proposition 4.5:}

If $\mathrm{A}$ and $\mathrm{B}$ be are zero commut idempotent matrices, then the following conditions are equivalent :

1) A-B is non-singular .

2) $\mathrm{N}(\mathrm{A}) \cap \mathrm{N}(\mathrm{B})=\{0\}$.

\section{Proof:}

$(1 \rightarrow 2)$ let $\overrightarrow{\mathbf{x}} \in \mathrm{N}(\mathrm{A}) \cap \mathrm{N}(\mathrm{B})$, then $\mathrm{A} \overrightarrow{\mathbf{x}}=0$ and $\mathrm{B} \overrightarrow{\mathbf{x}}=0$. So (A-B) $\overrightarrow{\mathbf{x}}=0$, therefore $\overrightarrow{\mathbf{x}} \in \mathrm{N}(\mathrm{A}-\mathrm{B})=0$ (since $\mathrm{A}-\mathrm{B}$ is non-singular). So $\overrightarrow{\mathbf{x}}=0$.

Hence $\mathrm{N}(\mathrm{A}) \cap \mathrm{N}(\mathrm{B})=\{0\}$.

$(2 \rightarrow 1)$ from [Proposition3.1] we get $\mathrm{N}(\mathrm{A}) \cap \mathrm{N}(\mathrm{B})=\mathrm{N}(\mathrm{A}-\mathrm{B})$, but $\mathrm{N}(\mathrm{A}) \cap \mathrm{N}(\mathrm{B})=\{0\}$, then $\mathrm{N}(\mathrm{A}-\mathrm{B})=\{0\}$.

Hence A-B is non-singular .

\section{References:-}

1) F. E. Hohn, "Introduction to linear algebra", The Macmillan Company, New York, (1972).

2) J. J. Koliha, V. Rakocevic and I. Straskraba, "The difference and sum of projectors", Linear Algebra Appl. 388(2004) ,279-288 .

3) J. J. Koliha, "Power bounded and exponentially bounded matrices", Applications of Math., 44(1999), 289-308 . 